\title{
Romanian Journal of Orthopaedic Surgery and Traumatology (ROJOST), a new journal in the Romanian academic environment
}

Romanian Journal of Orthopaedic Surgery and Traumatology (ROJOST) is an international, biannual newly established journal of the Romanian Society of Musculoskeletal Oncology (ROMSOS) and the Romanian Society of Arthroplasty (SROA), which aims to become the most important source of information for orthopedics, traumatologists, and researchers in the field.

High-level scientific articles, that address residents and specialists of orthopedics and traumatology, but also people who study, or want to be up to date with the latest news define the content of the journal.

The journal welcomes articles in the following categories: orientation articles, editorials, original works, clinical cases, subjects for residents, current medical education, and reviews that enhance the experience of physicians interested in this specialty.

The editorial board brings together nationally and internationally recognized academic personalities, who will contribute to the process of maintaining the journal at the highest standards. The journal benefits from an extensive team of physicians involved in the peer-review process, and will comply with the international rules of quality and originality, as far as the published articles are concerned.

RJOST is edited exclusively in English and "Carol Davila" University Press, in Bucharest, Romania, publishes its printed version.

The online version of ROJOST will be part of De Gruyter family, which will enhance the chances for future inclusion in Thomson Reuters/Clarivate Analytics, and other international databases.

For a proper publishing process, the journal will become member of CrossRef community, will use iThenticate for plagiarism detection, and, Editorial manager will be used for submitting and processing the articles. 